\title{
Resistance to extinction as a function of level of acquisition training and altered stimulus conditions during extinction
}

\author{
Donald Sehiff 1 \\ STATE UNIVERSITY COLLEGE, NEW PALTZ
}

\begin{abstract}
Rats given different amount of acquisition training (200, 400,800 or 1600 reinforcements) on a barpressing response and extinguished under varying degrees of stimulus change did not show any significantly different extinction curves from that of control Ss. Resistance to extinction and level of acquisition training was monotonically related in each extinction condition. However, reinstatement of the acquisition stimulus conditions led to significant extinction curves for the different groups.
\end{abstract}

\section{Problem}

In recent years, some doubt has been cast on the widely held assumption that resistance to extinction and number of continuously reinforced responses are monotonically related. There have been several reports (e. g., King, Wood, \& Butcher, 1961; North \& Stimmel, 1960) that resistance to extinction may be reduced with extensive acquisition training, though the relevant evidence is far from unequivocal (D'Amato, Schiff, \& Jagoda, 1962; Harris \& Nygaard, 1961).

One may ask why resistance to extinction and acquisition level are found to be monotonically related in some instances and not in other, similar, ones. An analysis of the King et al. study reveals that they did not adequately control for stimulus generalization decrement since Ss were extinguished under stimulus conditions which were somewhat altered from those of acquisition. More specifically, the length of the extinction session was considerably greater than the length of the usual acquisition session. It is conceivable that with extensive acquisition training Ss would come to discriminate the length of the daily training session. Consequently, for these Ss the latter portion of the extended extinction session would constitute a stimulus change which, due to stimulus generalization decrement, could result in a loss of response strength during that portion of the extinction session. Moderately trained Ss, on the other hand, should be less disturbed by such a stimulus alteration, since their opportunity to form a discrimination concerning session length is restricted. A similar analysis may be applied to other variables that undergo variation, advertently or inadvertently, from acquisition to extinction.

The present study was concerned with this problem and attempted to evaluate directly the effect of imposed stimulus changes on resistance to extinction after varying amounts of acquisition training. It was hypothesized that any stimulus alteration imposed during extinction would decrease resistance to extinction, and that the amount of this decrement would be a positive function of the amount of acquisition training. Thus, while allowing resistance to extinction and acquisition level to be monotonically related when no stimulus alterations occur during extinction, the present hypothesis implies a nonmonotonic relationship between these variables when stimulus alterations are introduced during extinction.

\section{Method}

The Ss were 184 albino rats, 80 to 100 days old. Two Grason-Stadler two-bar Skinner boxes were used; the right bars from each box were removed, converting them into single-bar boxes. Each box contained two pilot lights which served as stimulus sources.

Ss were randomly assigned to one of four acquisition levels $(200,400,800$ or 1600 reinforced bar-pressing responses). At the termination of acquisition training, Ss of each acquisition level were placed into one of three extinction groups. Extinction groups varied with respect to which stimulus component of the acquisition stimulus complex was absent during extinction.

Acquisition training followed three days of pretraining. Each S (22-hr. food deprived) made 50 rewarded bar presses per day until her required acquisition level was reached. Thus Ss of the 200, 400, 800 and 1600 groups required 4, 8, 16 and 32 training days, respectively.

Extinction lasted seven days. During the first six days, Ss of each acquisition level were extinguished with some component of the acquisition stimulus complex absent. More specifically, one group was extinguished in the absence of the two stimulus lights that were present during acquisition (Group L); a second group did not, during extinction, receive the click from the feeder mechanism (Group N). Finally, a third extinction group acted as a control. For this group, no stimulus change occurred during extinction.

On the seventh and last extinction day, the stimulus conditions of acquisition were reinstated for Group $\mathrm{L}$ and Group N.

\section{Results and Discussion}

The first six extinction days. The number of responses made by $S$ during each of the first six extinction days was converted to common logs, providing the basis for all statistical analyses.

The first question of concern is whether the extinction curves for the three extinction conditions are different. 


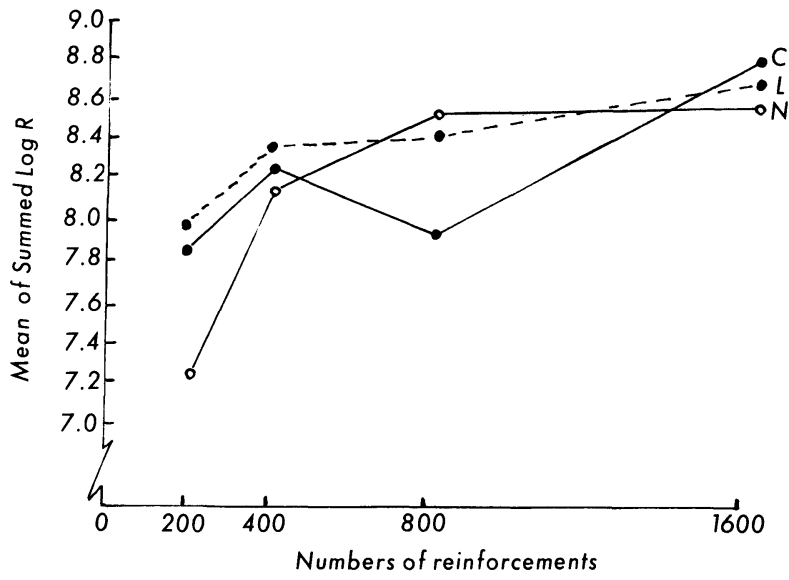

Fig. 1. Relationship between resistance to extinction and level of acquisition training for each of the.three extinction conditions.

Figure 1 shows the mean of the summed log. sources for the twelve extinction groups. Analysis of variance showed only acquisition levels to be significant $(F=7.9$; df, $3 / 172 ; p<.01)$. The results of a trend analysis revealed no significant differences among the linear and quadratic components of the three curves. As is apparent from Fig.1, all three curves are monotonic-a conclusion supported by trend analyses performed on each curve separately. Obviously, there is little difference among the functions produced by the three extinction conditions.

We next are concerned with whether the extinction groups followed a similar course of extinction over the

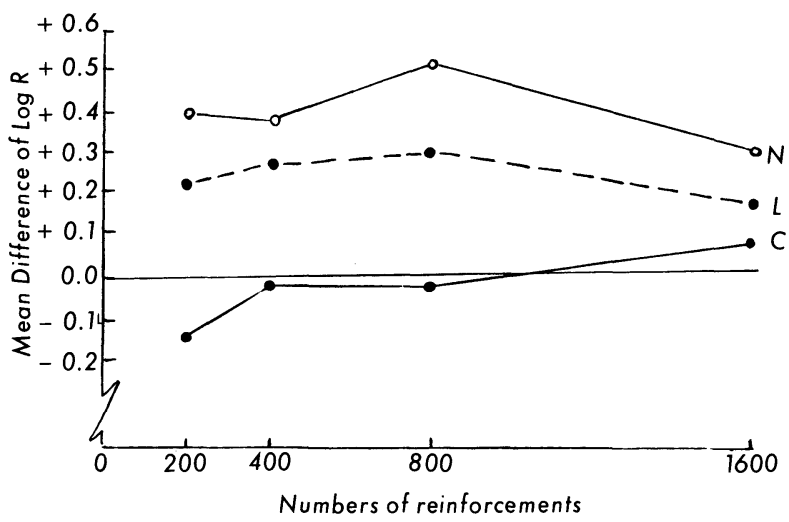

Fig. 2. Resistance to extinction on Day 7 in terms of difference scores (Day 7 - Day 6). six extinction days. A trend analysis was applied to these data which resulted in no significant differences. Thus, there is no evidence to indicate that alterations in the stimulus conditions prevailing during extinction had any effect on the course of extinction in the different groups.

The seventh extinction day. On the seventh and last extinction day, the stimulus conditions of acquisition were reinstated for all groups. Figure 2 shows the mean of the difference in log scores of Day 7 and Day 6. Positive values indicate, of course, an increase in responding on Day 7. Analysis of variance showed only extinction conditions to be significant, and well beyond the .001 level. Thus, although there is no evidence of a generalization decrement in Groups $\mathrm{L}$ and $\mathrm{N}$ during the first six extinction days, the importance of the light and click as cues for the bar-pressing response is clearly shown by the results of the seventh extinction day.

The findings of the present study do not support the hypothesis that resistance to extinction of a continuously reinforced response will be nonmonotonically related to acquisition level when stimulus elements are removed during extinction. Rather, they demonstrate that stimulus elements such as illumination level and magazine click may be relatively unimportant during normal extinction procedure. However, the results of the seventh extinction day (restitution of illumination level and magazine click) do indicate the importance of stimulus components present during acquisition. One implication of this finding is that a standard extinction procedure may not be an adequate test of the relative value of particular stimulus elements of the entire stimulus complex and that possibly a more sensitive test of their importance is the effects which occur when the stimulus elements are replaced.

\section{References}

D'AMATO, M. R., SCHIFF, D., \& JAGODA, H. Resistance to extinction after varying amounts of discriminative and non-discriminative instrumental training. J. exp. Psychol., 1962, 64, 526-532.

HARRIS, P., \& NYGAARD, J. E. Resistance to extinction and number of reinforcements. Psychol. Rep., 1961, 8, 233-234.

KING, R. A., WOOD, P., \& BUTCHER, J. Decreased resistance to extinction as a function of reinforcement. Amer. Psychologist, 1961, 16, 468. (Abstract)

NORTH, A. J., \& STIMMEL, D. T. Extinction of an instrumental response following a large number of reinforcements. Psychol. Rep., 1960, 6, 227-234.

Note

1. The author wishes to thank M. R. D'Amato for his help and encouragement during the course of this investigation. 